\title{
SPLINT THERAPY FOR TMJ CLICKING, WHICH TYPE IS MORE SUITABLE? A RANDOMIZED COMPARATIVE CLINICAL TRIAL
}

\author{
Mai A. Haggag* and Osama M.R. Askar **
}

\begin{abstract}
Purpose: Joint noises associated with temporomandibular joint (TMJ) internal derangement (ID) are often treated by using an intra-oral splint. The present study tried to find the most effective splint for this purpose, either full-arch maxillary stabilization splint (FAMSS) or anterior repositioning splint (ARS).
\end{abstract}

Materials and Methods: 50 patients (100 joints) with bilateral ID (presented with reciprocal clicking as the main complaint) were selected according to the Research Diagnostic Criteria for Temporomandibular Disorders (RDC/TMD). These patients were randomly and equally divided into two groups. Group I used FAMSS while Group II used ARS. Then, the sounds of both right and left TMJs were scored.

Results: Nearly all patients in both groups showed a decrease in the opening and closing sounds after 6 months. The reduction in TMJ clicking was highly significant in Group II (ARS) indicating a highly efficient splint therapy in managing joint clicking. Also, the study revealed a long-term stability of the gained results especially in Group II by following all patients for another 6 months without the usage of splints.

Conclusion: The results suggested that the use of 6-month ARS significantly reduced TMJ sounds with long-term positive effect so it can be the splint of choice for the management of TMJ clicking.

KEYWORDS: Full Arch Maxillary Stabilization Splint, Anterior Repositioning Splint, Anterior Disc Displacement with Reduction, TMJ Clicking, Temporomandibular Disorders.

\footnotetext{
* Assistant Professor of Oral and Maxillofacial Surgery, Faculty of Dentistry, Mansoura University
}

** Assistant Professor of Prosthodontics, Faculty of Dentistry, Mansoura University. 


\section{INTRODUCTION}

Temporomandibular joint internal derangement (TMJ ID) is considered as a developing degenerative condition at which the articular disc displaced anteriorly in relation to the condylar head pulling with it the retrodiscal tissue and remain in this location as long as the mouth is closed. Although during opening, the disc is trying to return to the original relation to the condylar head in order to allow mouth opening creating a clicking, popping or snapping sound which may be single or reciprocal that can be heard by the patient and in some times by the adjacent person. ${ }^{(1,2)}$

These sounds are considered as a clinical evidence and a diagnostic sign of disc displacement which may be occurred at any phase of mandibular opening, initial, middle or final phase. ${ }^{(3,4),(5)}$ The clicking sounds may or may not be accompanied with pain which can disturb the normal jaw functions which demand treatment. ${ }^{(6)}$

By reviewing the literature, there are several types of treatment appliances that can be used in the treatment of TMDs including the most widely used full arch stabilizing splint (Tanner appliance, Fox appliance, Michigan splint, or centric relation appliance) and anterior repositioning splint. ${ }^{(7-9)}$

Full arch maxillary stabilizing splint (FAMSS) is usually worn on the maxillary teeth. The mechanism of its action depends on the ability to provide a stable and ideal occlusion, eliminate occlusal prematurity, facilitate normal alignment between upper and lower teeth, decrease abnormal muscle movements and preventing teeth from clenching. (9-11) Another mechanism of action depends on the decompressive effect at the TMJ level which decreases the magnitude of joint loading. ${ }^{(12)}$

The other most commonly used appliance is anterior repositioning splint (ARS). It is known by its good effect in the treatment of cases complaining of anterior disc displacement with reduction (ADDwR) by moving the mandible to the therapeutic minimal protrusive position needed to eliminate the clinical signs which is mainly edge to edge position. ${ }^{(13)}$

The mechanism behind ARS action may be related to the anterior positioning of the mandible that allows the catch or re-capturing of the disc to the correct position in relation to the condylar head in the mandibular fossa as the disc-condyle complex walked back against the posterior slope of the articular eminence. ${ }^{(14,15)}$ Or the disc is slipped back to its normal relation to the condyle while the mandible is in its therapeutic position guided by ARS. ${ }^{(16)}$

Since one of the most characteristic symptoms accompanying with TMJ damage are abnormal joint sounds. This study was conducted to determine the most effective oral occlusal splint for the management of TMJ clicking which had been occurring for at least six months; either FAMSS or ARS. Since there is shortage in literatures relating to a standard therapeutic splint. The properties of time signs-symptoms occurred was critical in the selection of subjects.

The null hypothesis was that stabilizing splint does not differ from anterior repositioning splint in controlling symptomatic TMJ clicking either immediate post-insertion or 6 months after splint therapy.

\section{PATIENTS AND METHODS}

\section{Sample size:}

Using the nonparametric test (Friedman's test), the sample size was computed by adding $15 \%$ to the sample size required for the equivalent parametric test (one-way repeated measures ANOVA) based on the rule of thumb by Lehmann (1998). ${ }^{(17)}$ Assuming a medium effect size (partial h2 $=0.03$ ), a minimum sample size of 42 TMJs achieve a power of $82 \%$ with a significance level $(\alpha)$ of $5 \%$. By adding $15 \%$ to the required sample, a sample size of 50 TMJs ( 25 
patients) is required. So, our study was conducted on a total of 50 patients (100 TMJs) divided into two equal groups. The sample size was calculated by $\mathrm{G}^{*}$ Power software (version 3.1.9.7). ${ }^{(17)}$

Population: 50 adult patients with TMJ clicking were selected from Oral and Maxillofacial Surgery Department, Faculty of Dentistry, Mansoura University, Egypt.

All participants were fully informed about the study objective and signed a written consent form approved by the Ethics Committee (no. A12080921) and carried out in accordance with the Declaration of Helsinki. During the treatment period, all participants were instructed not to use any pharmacologic therapy.

All included patients have symptomatic disc displacement with reduction accompanied with clicking sounds as their main symptom. The diagnosis was based on RDC/TMD protocol (i.e., reproducible TMJ clicking sounds during jaw opening and closing with the opening click registered at $>5 \mathrm{~mm}$ interincisal distance and with TMJ clicking sounds that cannot be recognized when jaw opening is performed in a protruded position). Also, all selected patients should have class I dentition, without any other temporomandibular disorder.

Patients who had psychiatric disorders, pregnancy, systemic diseases, tumors, a history of TMJ surgery and/or previous TMDs treatment were excluded from the study.

Randomizing process: All included participants were randomized equally into two groups 25 patients each. The randomization was done with the aid of IBM-SPSS software (IBM Corp. Released 2019. IBM SPSS Statistics for Windows, Version 26.0. Armonk, NY: IBM Corp.) using randomized numbers before the study started.

Intervention: All participants were instructed not to eat a tough texture food and not trying to provoke TMJ clicking during the day. They used one of the following splints: [1] full hard stabilization splint (maxillary full coverage occlusal splints) in group I, [2] anterior repositioning splint (maxillary full coverage occlusal splints with an anterior ramp) in group II, The subjects were recalled after 1 week to check if the appliance was accepted and used.

Outcome: An objective scale for the recording of TMJ sounds was used. Reciprocal clicking was taken as a clinical sign of DDWR as it is consistently associated with it. ${ }^{(18,19)}$ The frequency of TMJ clicking sounds was stated on a 5-graded Scale(6) at each month's evaluation.

\begin{tabular}{cc}
\hline Scale & Criteria \\
\hline 0 & Never \\
\hline 1 & Occasionally \\
\hline 2 & Once or twice a month \\
\hline 3 & Once a week \\
\hline 4 & Several times a week \\
\hline 5 & Daily \\
\hline
\end{tabular}

\section{Construction of Splints (Fig.1):}

An alginate impression (Cavex Holland) of the maxillary and mandibular arches was made using automatic mixing machine with an adequate mixing ratio (Vmax. Alginate Mixer. Monitex Industrial. Taiwan), the impression was poured with a gypsum material (Durone IV, Dentsply) to have upper and lower casts. Facebow record (Elite Model facebowBio-Art. Brazil) was used to mount the upper cast on semi adjustable articulator (Bio-Art-A7 Plus. Brazil). A centric inter-occlusal record was made using lucia-jig in order to mount the lower cast in centric relation to the upper one. Protrusive and lateral records were made to adjust the protrusive and lateral condylar guidance's of the articulator. $3 \mathrm{~mm}$ vertical space was created between the upper and lower members of the articulator filled with the stent waxing up which was transferred to a clear heat cured acrylic resin. After flasking, finishing and polishing of the stent. It was returned back on the articulator for laboratory remount and check for occlusion. 

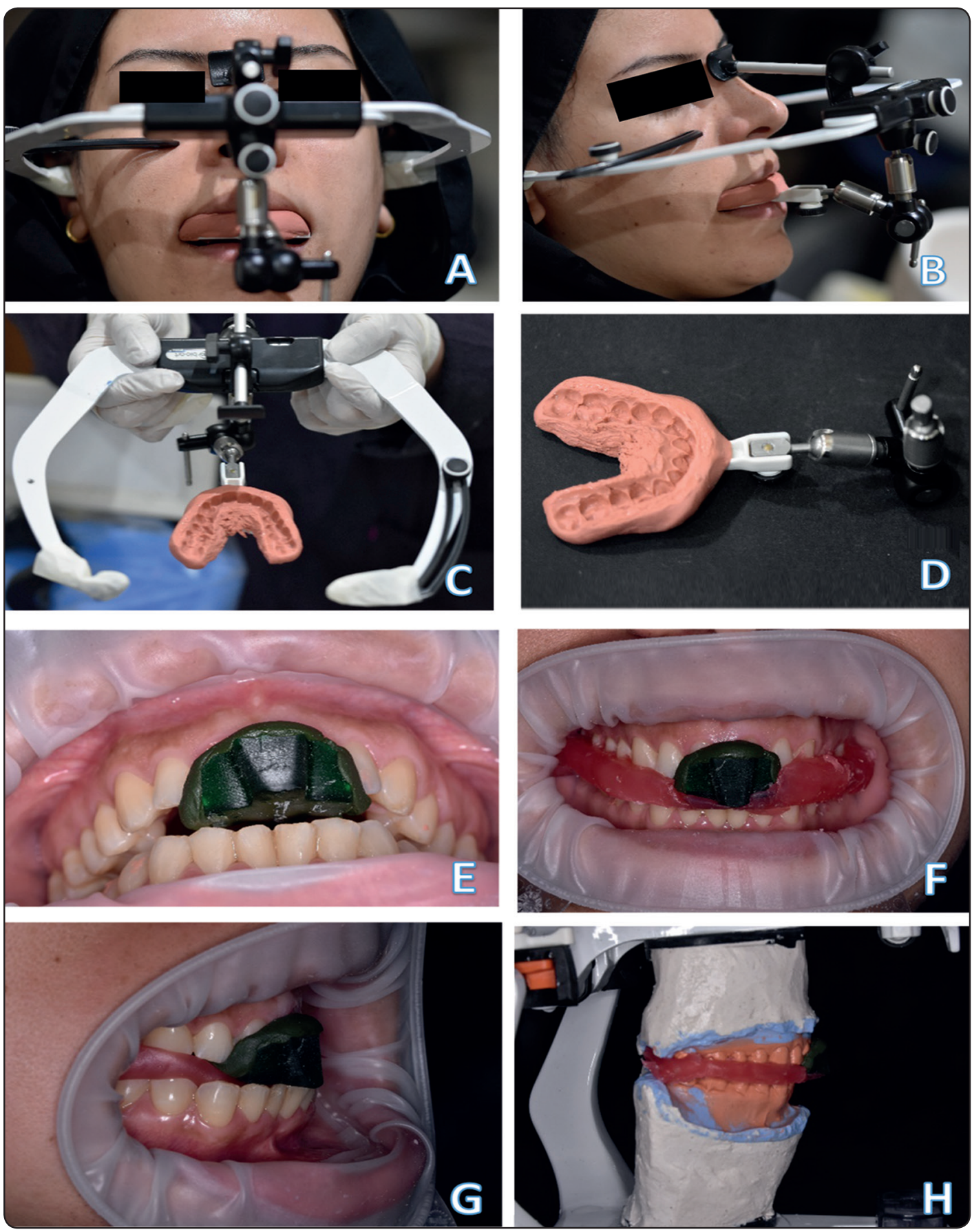

Fig. (1): Elite Facebow record; Frontal view (A). Lateral view (B). Facebow with registration bite (C). Silicon Bite fork attached to universal joint device of the Elite facebow (D). Lucia Jig (E). Lucia jig with centric relation record (F). Protrusive record (G). Mounted upper and lower casts on semi adjustable articulator $(\mathrm{H})$. 


\section{For the FAMS Splint group (Fig.2A):}

The wax pattern for FAMSS was adjusted to give balanced occlusal contact in centric occlusion and balanced articulation during eccentric lateral and protrusive movements, which reevaluated after processing of the stent and remounting. During delivery occlusion was re-checked again intraorally to produce even smooth and balanced articulation contact from centric occlusal position to eccentric lateral and protrusive excursions. The appliance was checked and adjusted continuously over the course of the therapy.

\section{For the AR Splint group (Fig.2B):}

The wax pattern for ARS was constructed with an anterior palatal ramp which guide the lower teeth smoothly to stop in a protrusive edge-to-edge relation between the lower and upper teeth without catching or locking of teeth, at the same time the posterior teeth showed no disclosing with full and even contact as described by Okeson. ${ }^{(20)}$ After processing and remounting, the appliance was rechecked intra orally. ${ }^{(21)}$ The click was disappeared after insertion which convinced and improved the patient confidence in the appliance.

\section{Subjects of both groups}

Wore the splints for $16 \mathrm{hrs} /$ day for 6 months. They were reviewed 1 month, 3 months and 6 months to confirm splint acceptance/compliance also to follow subjective treatment progress indicating short-term follow up periods, also reviewed at 6 months after treatment (without splint insertion) indicating longterm follow up period. ${ }^{(22)}$

\section{Statistical Analyses}

Data were entered and analyzed using IBMSPSS software (IBM Corp. Released 2019. IBM SPSS Statistics for Windows, Version 26.0. Armonk, NY: IBM Corp.). Qualitative data were expressed as absolute frequency $(\mathrm{N})$ and percentage (\%). Chi-Square was used to compare categorical data. Ordinal data (clicking scale) was expressed as median, interquartile range, and range. Repeatedly measured ordinal data in a group were compared by Friedman's test. Ordinal data between two groups were compared by Mann-Whitney U-test. Generalized estimating equations (GEE) test was run to ascertain the effect of two independent predictors [the treatment type (group) and the timing] on the likelihood that an ordinal dependent variable (clicking scale) will be higher. For any of the used tests, results were considered as statistically significant if $\mathrm{p}$ value $\leq 0.050$. Appropriate charts were used to graphically present the results whenever needed.

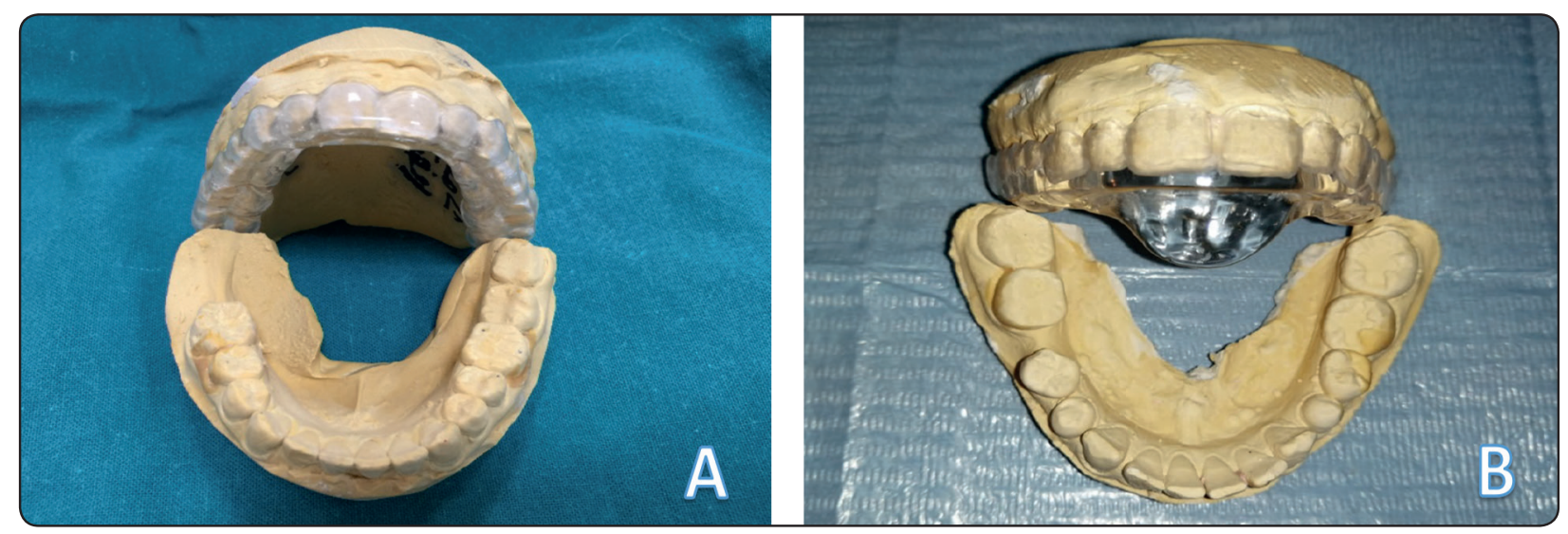

Fig. (2): Full Arch Maxillary Stabilizing Splint (A). Anterior Repositioning Splint (B). 


\section{RESULTS}

This study involved two age-, and sex-matched groups:

\section{Group I (Stabilizing Splint):}

This group involved 25 participants, 3 males (12\%), and 22 females (88\%). Their mean age (years) $\pm \mathrm{SD}$ is $23.2 \pm 6.8$ years.

\section{Group II (Anterior Repositioning Splint):}

This group involved 25 participants, 2 males $(8 \%)$, and 23 females (92\%). Their mean age (years) $\pm \mathrm{SD}$ is $23.5 \pm 6.7$ years.

There was no statistically significant difference in age (Independent-Samples t-test, $\mathrm{P}=0.868$ ), and sex (Fisher's exact test, $\mathrm{P}=1.000$ ) between the two groups.

5-graded Scale of TMJ clicking sounds: 3 : Once a week, 4: Several times a week, 5: Daily.
TABLE (1): Pretreatment scale in the two groups

\begin{tabular}{|c|c|c|c|c|}
\hline Scale & Group I & Group II & $c^{2}$ & $P$ value \\
\hline 3 & $7(14 \%)$ & $8(16 \%)$ & 0.331 & 0.847 \\
\hline 4 & $9(18 \%)$ & $7(14 \%)$ & & \\
\hline 5 & $34(68 \%)$ & $345(70 \%)$ & & \\
\hline
\end{tabular}

Notes: Data are N (\%). Test of significance is Chi-Square test.

This table shows comparable proportions of each of the three scales in each group at enrollment. (Tab.1)

Table (2) shows a statistically significant difference in the scale between the 5-time points in each group.

In group I Pairwise comparisons showed a statistically significant difference between pretreatment values as well as one-month values vs. 3-, 6-, and 12-months values. While there was no difference between pretreatment and one-month values, and between 3-, 6-, and 12-months values.

TABLE (2): Scale over time in each of the two groups

\begin{tabular}{|c|c|c|c|c|c|c|c|}
\hline Group & Pretreatment & 1-month & 3-months & 6-months & 12-months & $\chi^{2}[4]$ & Pvalue \\
\hline Group I & & & & & & 132.41 & $<0.001$ \\
\hline Median & 5 & 4 & 3 & 2 & 3 & & \\
\hline $25^{\text {th }}$ percentile & 4 & 3 & 2 & 2 & 2 & & \\
\hline $75^{\text {th }}$ percentile & 5 & 5 & 4 & 3 & 4 & & \\
\hline Minimum & 3 & 0 & 0 & 0 & 0 & & \\
\hline Maximum & 5 & 5 & 4 & 4 & 5 & & \\
\hline Pairwise & A & A & B & B & B & & \\
\hline Group II & & & & & & 143.85 & $<0.001$ \\
\hline Median & 5 & 2 & 1 & 0 & 1 & & \\
\hline $25^{\text {th }}$ percentile & 4 & 1 & 0 & 0 & 0 & & \\
\hline $75^{\text {th }}$ percentile & 5 & 3 & 2 & 1 & 1 & & \\
\hline Minimum & 3 & 0 & 0 & 0 & 0 & & \\
\hline Maximum & 5 & 4 & 3 & 2 & 5 & & \\
\hline Pairwise & A & B & $\mathrm{C}$ & $\mathrm{C}$ & $\mathrm{B}, \mathrm{C}$ & & \\
\hline
\end{tabular}

Notes: Test of significance is Friedman's test. Pairwise comparisons are in letters, similar letters = Insignificant difference, different letters $=$ Significant difference . 
In group II Pairwise comparisons revealed a statistically significant difference between pretreatment values vs. all post-treatment values, these results verified the early positive effect of ARS. Also, it revealed a statistically significant difference between one-month values vs. all 3-, and 6-months post-treatment values (that showed continued improvement) but not 12-month values. There was no difference between 3-, 6-, and 12-months values. Moreover, it showed long-term stability of the gained improvement. (Tab.2)

Table (3): Scale at each time point in the two groups:

\begin{tabular}{ccccc}
\hline Timing & Group I & Group II & Z value & P value \\
\hline Pretreatment & $5(4-5)$ & $5(4-5)$ & -0.114 & 0.909 \\
\hline One-month & $4(3-5)$ & $2(1-3)$ & -6.973 & $<\mathbf{0 . 0 0 1}$ \\
\hline Three-months & $3(2-4)$ & $1(0-2)$ & -5.676 & $<\mathbf{0 . 0 0 1}$ \\
\hline Six-months & $2(2-3)$ & $0(0-1)$ & -7.317 & $<\mathbf{0 . 0 0 1}$ \\
\hline Twelve-months & $3(2-4)$ & $1(0-1)$ & -5.574 & $<\mathbf{0 . 0 0 1}$ \\
\hline
\end{tabular}

Notes: Data are median $\left(25^{\text {th }}\right.$ percentile $-75^{\text {th }}$ percentile $)$.

Test of significance is Mann-Whitney $U$-test.

By comparing the two groups, table (3) shows a statistically significant lower scale in group II vs. group I at each post-treatment time points but not in the pre-treatment values which indicates a high positive effect of ARS that used in group II.

Generalized estimating equations (GEE) was run to ascertain the effect of two independent predictors, the treatment type (group) and the timing on the likelihood that the clicking scale (ordinal dependent variable) will be higher. (Tab.4)

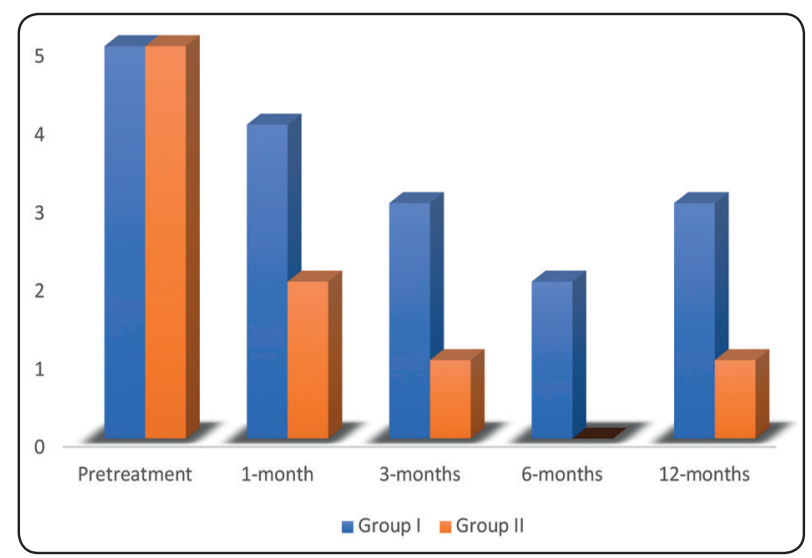

Graph (1): Scale at each time point in the two groups

Parameter estimates show that group II vs. group I has a decreased probability of being in a higher level of clicking scale (odds ratio $=0.137$ ). Parameter estimates also show a decreasing probability of being in a higher level of clicking scale as time increases (odds ratio $=0.387$ ). $($ Tab.4)

\section{DISCUSSION}

Women showed increased incidence of TMJ disorders than men. ${ }^{(23)}$ This may be related to several factors like biomechanical, physiological, genetic, and hormonal factors. ${ }^{(24)}$ The presence of estrogen receptors in women's joints may change the metabolic functions and rises ligament laxity which hypothesized to be related to increase TMJ disorders in females. ${ }^{(25)}$ This was in agreement with our study sample where women represented the majority.

The present study depended mainly on clinical signs and symptoms, this is supported by Talaat WM et al ${ }^{(26)}$ who stated that DDWR is a highly prevalent

TABLE (4): Parameter estimates of generalized estimating equations (GEE) for predicting the clicking scale

\begin{tabular}{lcccc}
\hline Predictor & LR $\chi^{2}[\mathbf{1}]$ & Wald $\chi^{2}[\mathbf{1}]$ & P value & OR (95\% CI for OR) \\
\hline Group II vs. group I & 120.029 & 1124.671 & $<\mathbf{0 . 0 0 1}$ & $0.137(0.097-0.195)$ \\
\hline Increasing time & 146.958 & 172.198 & $<\mathbf{0 . 0 0 1}$ & $0.387(0.336-446)$ \\
\hline
\end{tabular}

Notes: LR = Likelihood Ratio. 
clinical condition, various literatures report that most of general population, possibly $90 \%$, shows mild disc displacements similar to those of patients seeking treatment. About $1 / 3$ of them were asymptomatic (without pain or dysfunction) and had documented TMJ disc displacement. Also Marpaung CM et al (27) stated that the clinical TMJ assessment can be considered a benchmark for DDWR diagnosis; as DDWR only becomes clinically evident when it interferes with TMJ function.

According to many studies ${ }^{(1,28)}$, joint sounds and associated pain are eliminated once the disc recaptured by the condyle allowing smooth, coordinated painless range of motion. Which is the major goal of our study. The chance of disc 're-capture' is stated to depend on many factors such as; disc-condyle position and configuration, posterior attached tissues integrity and the degree of degenerative changes in the TMJ. ${ }^{(29)}$ Therefore, only patients with recently occurring joint click and not complaining from any other TMD were included in the present study.

In agreement with the results of the present study, Several studies suggested that ARS is superior in managing TMJ sounds..$^{(1,28,30)}$ Lundh $H$ WP et al stated that AR splint is superior to the flat occlusal splint in decreasing or removing clicking, joint pain, and accompanying muscular symptoms. ${ }^{(14)}$

Also, Al-Moraissi EA et al revealed that ARS and counselling therapy significantly reduced the incidence of TMJ clicking when compared to a full hard stabilization splint.(7) As well, other studies have stated its superiority over other occlusal splint designs for the management of ADDwR. ${ }^{(1,31,32)}$ There is a lack of studies that showed superiority of flat occlusal splint over AR splint in the management of TMJ clicking.

Tecco $S$. et al $^{(1)}$ stated that the occurrence of joint noises reduced over time and the AR splint appears not to make any change in joint noises. But they also suggested that AR splint might be superior to FAMS splint in case of recently occurring sounds.$^{(1)}$ which agreed with the results of the present study as all the included subjects were complaining of a recently occurring click.

In accordance with the results of the present study, Ma Z et al revealed that TMJ clicking was significantly reduced in the treated subjects and this also agreed with the findings of Fayed et al ${ }^{(33)}$ and Simmons and Gibbs $^{(34)}$, who stated that the regaining of a harmonious relationship within TMJ might be the reason behind the elimination of clicking.

The present study revealed highly accepted results in ARS group during different follow up periods and also after 6 months without using the splint indicating long-term positive effect, that may be due to the morphologic changes and remodeling of the joint structures over time by reducing the physical obstacle of the condyle translation as mentioned by Kaymak D et al. ${ }^{(35)}$

Moreover, the stoppage of TMJ over-loading and the associated progressive adaptation of the joint structures could be considered as the cause of the long-term positive effect that in turn reduce the joint sounds. According to that, the longer time of using an ARS could provide a more reduction in the clicking sounds due to longer adaptation period. ${ }^{(35)}$

The current study showed much more favorable results than the study of Kaymak D et al ${ }^{(35)}$ who revealed only a reduction in TMJ sounds using 6 weeks-ARS. This may be due to a longer use of ARS (6 months) in the present study.

In contrary to the results of the present study, many studies revealed recurrence of joint sounds. This indicated that disc 're-capture' wasn't permanent in the majority of treated individuals. $(1,31,36)$ Others reported that only during splint therapy, the normal condyle-disc relationship can be maintained. Also, they stated that the intensity of joint sounds could only be reduced but couldn't be eliminated completely. ${ }^{(28,37)}$ Moreover Chen HM et al showed a positive short-term effect of a 3-months ARS that used only during sleep with a lower longterm usefulness. ${ }^{(22)}$ 
The present study used edge-to-edge position to fabricate the ARS as the favorable therapeutic mandibular position. As ARS with edge-toedge position resulted in nearly $100 \%$ disc recapture which is proved by MRI and this position is well tolerated by the patients. Also, recurrent locking and/or symptomatic joint clicking have been eliminated. After splint insertion, there was insignificant difference in disc-condyle angles between individuals either with ADDwR or with normal joints. ${ }^{(29)}$

Also, Chen HM FK et al stated that, only $54.8 \%$ disc recapture was obtained in the least protruded position using MRI. But, ARS with edge-to-edge relation showed increase the Success rates to $93.5 \% .^{(38)}$

\section{CONCLUSION}

Lately, the number of patients complaining only of TMJ clicking without any other TMDs were greatly increased. This may be due to the increased cognition of population about TMDs and there sequala. The null hypothesis was rejected which mean that the use of 6-month edge to edge ARS significantly reduced TMJ sounds with a long-term positive effect and can be considered as the splint of choice in this situation.

\section{Conflict of Interest}

Nil.

\section{REFERENCE}

1. Tecco S, Festa F, Salini V, Epifania E, D’Attilio M. Treatment of joint pain and joint noises associated with a recent TMJ internal derangement: a comparison of an anterior repositioning splint, a full-arch maxillary stabilization splint, and an untreated control group. Cranio. 2004; 22(3):209-19.

2. AL Y. Internal derangements of the temporomandibular joint: A review of the anatomy, diagnosis, and management. J Indian Prosthodont Soc. 2015;15:2-7.

3. Huang ZS LX,LiXL.Characteristics of temporomandibular joint vibrations in anterior disk displacement with reduction in adults. Cranio. 2011;29:276-83.
4. Sano T WS, Westesson PL, et al. Amplitude and frequency spectrum of temporomandibular joint sounds from subjects with and without other signs/symptoms of temporomandibular disorders. J Oral Rehabil. 1999; 26: $145-50$.

5. Pihut M, Gorecka M, Ceranowicz P, Wieckiewicz M. The Efficiency of Anterior Repositioning Splints in the Management of Pain Related to Temporomandibular Joint Disc Displacement with Reduction. Pain Res Manag. 2018:9089286.

6. Wanman A, Marklund S. Treatment outcome of supervised exercise, home exercise and bite splint therapy, respectively, in patients with symptomatic disc displacement with reduction: A randomised clinical trial. J Oral Rehabil. 2020; 47(2):143-9.

7. Al-Moraissi EA, Farea R, Qasem KA, Al-Wadeai MS, Al-Sabahi ME, Al-Iryani GM. Effectiveness of occlusal splint therapy in the management of temporomandibular disorders: network meta-analysis of randomized controlled trials. Int J Oral Maxillofac Surg. 2020;49(8):1042-56.

8. Fricton $\mathrm{J}$ LJ, Wright $\mathrm{E}$, et al. . Systematic review and meta-analysis of randomized controlled trials evaluating intraoral orthopedic appliances for temporomandibular disorders. J Orofac Pain 2010;24:237-54.

9. Gray RJM DS, Quayle AA. Temporomandibular Disorders: A Clinical Approach. London: British Dental Association; 1995.

10. Miralles R ZP, Santander H, Manns A. Influence of occlusal splints on bilateral anterior temporal EMG activity during swallowing of saliva in subjects with craniomandibular dysfunction. J Craniomandib Pract 1991; 9:129-36.

11. Truelove E HK, Mancl L, Dworkin SF. . The efficacy of traditional, low-cost and nonsplint therapies for temporomandibular disorder: a randomized controlled trial. JADA. 2006;137:1099-107.

12. Dao TT, Lavigne GJ. Oral splints: the crutches for temporomandibular disorders and bruxism? Crit Rev Oral Biol Med. 1998;9(3):345-61.

13. HC. S. Guidelines for anterior repositioning Appliance therapy for the management of craniofacial pain and TMD. Cranio 2005; 23:300-5.

14. Lundh H WP, Kopp S, Tillstrom B. . Anterior repositioning splint in the treatment of temporomandibular joints with reciprocal clicking: comparison with a flat occlusal splint and an untreated control group. . Oral Surg Oral Med Oral Pathol 1985; 60:131-6. 
15. Mosby; TjammdneIZGeSL. 1994:541-2.

16. Kurita H KK, Ohtsuka A, Kotani A. . Change of position of the temporomandibular joint disk with insertion of a diskrepositioning appliance. Oral Surg Oral Med Oral Pathol Oral Radiol Endod 1998;85:142-5.

17. Erich L. Lehmann: Nonparametrics: Statistical Methods Based on Ranks R, 1998, ISBN=978-0139977350, pages 76-81.

18. Eriksson L WP-L, Rohlin M. Temporomandibular joint sounds in patients with disk displacement. Int J Oral Surg 1985;14:428-36.

19. Rohlin M WP-L, Eriksson L. The correlation of temporomandibular joint sounds with joint morphology in 55 autopsy specimens. J Oral Maxillofac Surg. 1985; 43:194-200.

20. Okeson JP: Management of temporomandibular disorders and occlusion. 3rd ed. St Louis: CV Mosby. 1993:477-9.

21. Karakis D DA, Bek B. Evaluation of the effect of two different occlusal splints on maximum occlusal force in patients with sleep bruxism: A pilot study. J Adv Prosthodont 2014;6:103-8.

22. Chen HM, Liu MQ, Yap AU, Fu KY. Physiological effects of anterior repositioning splint on temporomandibular joint disc displacement: a quantitative analysis. J Oral Rehabil. 2017; 44(9):664-72.

23. Ma Z, Xie Q, Yang C, Zhang S, Shen Y, Abdelrehem A. Can anterior repositioning splint effectively treat temporomandibular joint disc displacement? Sci Rep. 2019; 9(1):534

24. BadelT,Marotti,M.,Kern,J.\& Laskarin,M. .Aquantitative analysis of splint therapy of displaced temporomandibular joint disc. Ann Anat 2009;191: 280-7.

25. Chisnoiu AMea. Factors involved in the etiology of temporomandibular disorders - a literature review. . Clujul Med 2015;88:473-8.

26. Talaat WM, Adel OI, Al Bayatti S. Prevalence of temporomandibular disorders discovered incidentally during routine dental examination using the Research Diagnostic Criteria for Temporomandibular Disorders. Oral Surg Oral Med Oral Pathol Oral Radiol. 2018; 125(3): 250-9.

27. Marpaung CM, Kalaykova SI, Lobbezoo F, Naeije M. Validity of functional diagnostic examination for temporomandibular joint disc displacement with reduction. J Oral Rehabil. 2014;41(4):243-9.
28. Conti PC CA, Lauris JR, Stuginski-Barbosa J. Management of painful temporomandibular joint clicking with different intraoral devices and counseling: A controlled study. J Appl Oral Sci 2015;23:529-35.

29. Eberhard D BH, Steger W. The efficacy of anterior repositioning splint therapy studied by magnetic resonance imaging. Eur J Orthod 2002;24:343-52.

30. Hersek N UG, Cindas A, Canay S, Kutsal YG. Effect of anterior repositioning splints on the electromyographic activities of masseter and anterior temporalis muscles. Cranio. 1998; 16:11-6.

31. Zamburlini I AD. Long-term results of appliance therapies in anterior disk displacement with reduction: a review of the literature. Cranio 1991; 9:361-8.

32. Tecco S CS, Teté S, Orsini G, Festa F. Intra-articular and muscle symptoms and subjective relief during TMJ internal derangement treatment with maxillary anterior repositioning splint or SVED and MORA splints: A comparison with untreated control subjects. Cranio. 2006; 24:119-29.

33. Fayed MM, El-Mangoury, N. H., El-Bokle, D. N. \& Belal, A. I. Occlusal splint therapy and magnetic resonance imaging. World J Orthod 2004;5:133-40

34. Simmons HCrG, S. J. Recapture of temporomandibular joint disks using anterior repositioning appliances: an MRI study. Cranio 1995;13: 227-37

35. Kaymak D, Karakis D, Dogan A. Evolutionary Spectral Analysis of Temporomandibular Joint Sounds Before and After Anterior Repositioning Splint Therapy in Patients with Internal Derangement. Int J Prosthodont. 2019; 32(6): 475-81.

36. JP. O. Long-term treatment of disk-interference disorders of the temporomandibular joint with anterior repositioning occlusal splints. . J Prosthet Dent 1988;60:611-6.

37. Conti PC MJ, Conti AC, Pegoraro LF, Araújo Cdos R. Partial time use of anterior repositioning splints in the management of TMJ pain and dysfunction: A one-year controlled study. . J Appl Oral Sci 2005;13:345-50.

38. Chen HM FK, Li YW, Zhang ZK. Positional changes of temporomandibular joint disk and condyle with insertion of anterior repositioning splint. Hua Xi Kou Qiang Yi Xue Za Zhi. 2009; 27(4): 408-412.

39. Positional changes of temporomandibular joint disk and condyle with insertion of anterior repositioning splint. Hua Xi Kou Qiang Yi Xue Za Zhi 2009;27: 408-12. 